\title{
DYNAMICAL AGENTS' STRATEGIES AND THE FRACTAL MARKET HYPOTHESIS
}

\author{
Lukáš VÁCHA, Miloslav S. VOŠVRDA*
}

\begin{abstract}
:
The efficient market hypothesis $(E M H)$ fails as a valid model of financial markets. The fractal market hypothesis (FMH) is a more general alternative way to the EMH. The FMH is formed on the following parameter space: agents' investment horizons. A financial market is more stable when a fractal character in the structures of agent's investment horizons is adopted. For computer simulations, the classical model is modified. This adjusted model shows that various frequency distributions on agents' investment horizons lead to different returns behaviour. The FMH focuses on matching of demand and supply of agents' investment horizons in the financial market. The FMH asserts that investors have different information based on temporal attributes. Since all investors in the market have different time investment horizons, the market remains stable. Our simulations of probability distributions of agents' investment horizons demonstrate that many investment horizons guarantee stability on the financial market.
\end{abstract}

Keywords: efficient market hypothesis, fractal market hypothesis, agents' investment horizons, agents' trading strategies

JEL Classification: C61, G14, D84

\section{Introduction}

The $\mathrm{EMH}$ was a paradigm of economic and finance theory for the last twenty years. After empirical data analysis on financial markets and after theoretical economic and finance progress this paradigm is gotten over. There are phenomena observed in real data collected from financial markets that cannot be explained by the recent economic and finance theories. One paradigm of recent economic and finance theory claims that sources of risk and economic fluctuations are exogenous. Therefore the economic system would converge to a steady-state path, which is deter-

*) Institute of Information Theory and Automation of the Academy of Sciences of the Czech Republic, Pod Vodárenskou věží 4, CZ - 18208 Prague 8 (e-mail: Ivacha@ @eznam.cz; vosvrda@utia.cas.cz ).

${ }^{\star *}$ ) A financial support from the Grant Agency of Charles University, Prague under the grant No. 454/ 2004/A EK/FSV and from the Grant Agency of the CR under the grant No. 402/03/H057 and Metrostav a.s. is gratefully acknowledged. 
mined by fundamentals and there are no opportunities for speculative profits in the absence of external shocks prices. It means that the other factors play an important role in the construction of real market forces as heterogeneous expectations. Since agents do not have sufficient knowledge of the structure of the economy to form correct theoretical expectations, it is impossible for any formal theory to postulate unique value expectations that would be held by all agents (see Gaunersdorfer, 2000). Prices are partly determined by fundamentals and partly by the observed fluctuations endogenously caused by non-linear market forces. This implies that technical trading rules need not be systematically bad and may help in predicting future price changes. Developments in the theory of non-linear dynamic systems have contributed to the new approaches in economics and finance theory (see Brock, 2001). Introducing non-linearity in these models may improve research of a mechanism generating the observed movements in the real financial data. The financial market exhibits local randomness but also global determinism. Thus financial markets can be considered as non-linear dynamic systems of the interacting agents processing new information immediately. Investors with the same investment horizons, and holding similar positions in the market may utilize this information differently. Therefore a financial market has a fractal structure in investment horizons. For analysing of behaviour of such market, an adjusted version of the model, introduced by Vácha and Vošvrda (see Vošvrda, 2001) with two main types of traders, i.e. fundamentalists and technical traders, is used. Technical traders tend to put little faith in strict efficient markets. Fundamentalists rely on their model employing fundamental information basis for forecasting the next price period. The traders determine whether current conditions call for the acquisition of fundamental information in a forward looking manners, rather than relying on post performance. This approach relies on heterogeneity in the agent information and subsequent decisions either as fundamentalists or as chartists. Changing the chartist's profitability and fundamentalist's positions is a basis of the cycles behaviour. A more detailed analysis bas been introduced in the Brock and Hommes model. This model with memory was analysed (see Tu, 1992). The model is presented in a form of evolutionary dynamics of the price model. The fundamentalists are considered as traders with more rich structure of memory for a price prediction. The chartists are considered as traders with more simple structure of memory for the price prediction. A simulation analysis of this model under changing probability properties of memory shows connections between $\mathrm{EMH}$ and $\mathrm{FMH}$. Section 2 is devoted to dynamics of fractions of different traders. Agent's investment horizons with a different form of memory structures in the performance measure are analysed. Fractal structure of financial markets is shown in Section 3. Results of the simulation analysis are introduced in Section 4.

\section{Dynamics of Traders}

Let us concentrate on dynamics of the fractions $n_{h, t}$ of different $h$-trader types, i.e.

$$
a \cdot x_{t}=\sum_{h} n_{h, t-1} \cdot f_{h}\left(x_{t-1}, \ldots, x_{t-L}\right) \equiv \sum_{h} n_{h, t-1} \cdot f_{h, t},
$$

where $n_{h, t-1}$ denotes the fraction of trader type $h$ at the beginning of period $t$, before than the equilibrium price $x_{t}$ has been observed and a denotes a gross return of a risk free asset which is perfectly elastically supplied, i.e., $a>1$ and $L$ is a number of lags. Now the realized excess return $Z_{t+1}$ over period $t$ to the period $t+1$ is computed, where $x_{t}=p_{t}-p_{t}^{*}$, and $p_{t}^{*}$ is the price corresponding to the intersection point of demand and supply, by 


$$
\begin{gathered}
Z_{t+1}=p_{t+1}-a \cdot p_{t}, \\
Z_{t+1}=x_{t+1}+p_{t+1}^{*}-a \cdot x_{t}-a \cdot p_{t}^{*}, \\
Z_{t+1}=x_{t+1}-a \cdot x_{t}+p_{t+1}^{*}-E_{t}\left(p_{t+1}^{*}\right)+E_{t}\left(p_{t+1}^{*}\right)-a \cdot p_{t}^{*}
\end{gathered}
$$

From the equation (4) we get

$$
E_{t}\left(p_{t+1}^{*}\right)-a \cdot p_{t}^{*}=0 \text { and } \delta_{t+1}=p_{t+1}^{*}-E_{t}\left(p_{t+1}^{*}\right),
$$

note that $(5)$ is a martingale difference sequence with respect to $\mathfrak{I}_{t}$ i.e.,

$$
E_{t}\left(\delta_{t+1} \mid \mathfrak{I}_{t}\right)=0
$$

for all $t$. So Eq. (4) can be written as follows

$$
Z_{t+1}=x_{t+1}-a \cdot x_{t}+\delta_{t+1} \text {. }
$$

The decomposition of the equation (6) is separating the 'explanation' part of realized excess returns $Z_{t+1}$ into the contribution $x_{t+1}-a \cdot x_{t}$ and the additional part $\delta_{t+1}$. Let a performance measure $\pi\left(Z_{t+1}, \rho_{h, t)}\right.$ be defined by

$$
\pi_{h, t}=\pi\left(Z_{t+1}, \rho_{h, t}\right)=Z_{t+1} \cdot z_{t} \cdot \rho_{h, t} \pi_{h, t}=\left(x_{t+1}-a \cdot x_{t}+\delta_{t+1}\right) \cdot z_{t} \cdot \rho_{h, t}
$$

where

$$
\rho_{h, t}=E_{h, t}\left(Z_{t+1}\right)=f_{h, t}-a \cdot x_{t}
$$

and $z_{t}$ denotes the number of shares of the asset purchased at time $t$. So the $h$-performance is given by the realized profits for the $h$-trader. Let the updated fractions $n_{h, t}$ be given by the discrete choice probability

$$
\begin{aligned}
n_{h, t} & =\exp \left(\beta \cdot \pi_{h, t-1}\right) / Y_{t}, \\
Y_{t} & =\sum_{h} \exp \left(\beta \cdot \pi_{h, t-1}\right) .
\end{aligned}
$$

The parameter $\beta$ is the intensity of choice measuring how fast agents switch between different predictors. The parameter $\beta$ is a measure of trader's rationality. The variable $Y_{t}$ is just a normalization so that the fractions $n_{h, t}$ sum up to 1 . If the intensity of choice is infinite $(\beta=+\infty)$, the entire mass of traders uses the strategy that has the highest fitness. If the intensity of choice is zero, the mass of traders distributes itself evenly across the set of available strategies.

\section{Memory in the Performance Measure}

The performance measure is given by summation of $m$-values of the lagged $h$ performance measures in the following form

$$
\begin{aligned}
& n_{h, t}=\exp \left(\beta \cdot \frac{1}{m} \sum_{p=1}^{m} \pi_{h, t-p}\right) / Y_{t}, \\
& Y_{t}=\sum_{h} \exp \left(\beta \cdot \frac{1}{m} \sum_{p=1}^{m} \pi_{h, t-p}\right),
\end{aligned}
$$

where the $m$ denotes the memory length, and the $\eta$ is the realization of the random vector of predictor memory (trading horizons). We assume that the expression $m=E_{h}[\eta]$ holds. All beliefs or the formation of expectations with different lag lengths will be of the following form 


$$
f_{h, t}=g_{h} \cdot x_{t-1}+b_{h}
$$

where $g_{h}$ denotes the trend, $b_{h}$ the bias of trader type $h$. If $b_{h}=0$, the agent $h$ is called a pure trend chaser if $g_{h}>0$ (strong trend chaser if $g_{h}>a$ ) and a contrarian if $g_{h}<0$ (strong contrarian if $g_{h}<-a$ ). If $g_{h}=0$, type $h$ trader is said to be purely biased. He is upward (downward) biased if $b_{h}>0\left(b_{h}<0\right)$. In the special case $g_{h}=b_{h}=0$, type $h$ trader is called fundamentalist, i.e. the trader believes that prices return to their fundamental value. Fundamentalists do have all the past prices and dividends in their information set, but they do not know the fractions $n_{h, t}$ of the other belief types.

\section{Range Scale Analysis of Agent Investment Stra- tegies}

For estimating and analysing the correlation structures on capital markets, a nonparametric method of Hurst type is used. $\mathrm{H}$. E. Hurst discovered very robust nonparametric methodology which is called rescaled range analysis, or $R / S$ analysis. The $R / S$ analysis was used for distinguishing random and non-random systems, the persistence of trends, and duration of cycles. This method is very convenient for distinguishing random time series from fractal time series as well. Starting point for the Hurst's coefficient was the Brownian motion as a primary model for random walk processes.

For computation of the $R / S$ coefficients we have to divide the analyzed time series $\sum_{i=1}^{T} X_{i}$ into $N$ contiguous subintervals of the length $n$. Each subinterval $\chi_{k}$. with $k=1,2, \ldots, N$. Each element in $\chi_{k}$ is labeled by $T_{j, k}$ such that $j=1,2, \ldots, n$. For each $\chi_{k}$ of length $n$, the mean value is $\bar{X}_{k}=\frac{1}{n} \sum_{j=1}^{n} T_{j, k}$. The time series of accumulated departures $\left\{X_{j, k}\right\}_{j=1}^{n} N_{k=1}^{N}$ from mean values for each subinterval $\chi_{k}$ is $X_{j, k}=\sum_{i=1}^{j}\left(T_{i, k}-\bar{X}_{k}\right)$ for $j=1,2, \ldots, n$. For given $n$, the values $(R / S)_{n}$ is defined in the following form

where

$$
(R / S)_{n}=\frac{1}{N} \sum_{k=1}^{N} \frac{R_{x_{k}}}{S_{x_{k}}}
$$

$$
R_{\chi k}=\max _{1 \leq j \leq n}\left[X_{j, k}\right]-\min _{1 \leq j \leq n}\left[X_{j, k}\right]
$$

is the range for each subinterval $\chi_{k}$ and

$$
S_{\chi k}=\sqrt{\frac{1}{n} \sum_{j=1}^{n}\left(T_{j, k}-X_{k}\right)^{2}}
$$

is the sample standard deviation for each subinterval $\chi_{k}$. The Hurst exponent $H$ can be approximated as well by the following equation

$$
\log \left((R / S)_{n}\right)=\log (c)+H \log (n)
$$

where $c$ is a constant. If a system of random variables $\left\{(R / S)_{n}\right\}$ is i.i.d, then $H=0.5$. The values of the Hurst exponent belonging to $0<H<0.5$ signifies antipersistent system of variables covering less space than random ones. Such a system must reverse itself more frequently than a random process. With the assumption of a 
stable mean (which we do not impose here) we can equate this behaviour to a meanreverting process. The values $0.5<H<1$ show persistent process that is characterized by long memory effects. This long memory occurs regardless of the time scale, i.e. there is no characteristic time scale which is the key characteristic of fractal time series (see Peters, 1994). For obtaining the expected $\left\{(R / S)_{n}\right\}$ values for i.i.d. process we have used the following equation (see Peters, 1994):

$$
E(R / S)_{n}=\left(\frac{n-0.5}{n}\right)\left(n \cdot \frac{\pi}{2}\right)^{-\frac{1}{2}} \cdot \sum_{r=1}^{n-1} \sqrt{\frac{n-r}{r}}
$$

Moreover, we have used the $V$-statistic which estimates the breaks in the $R / S$ plot in an easier way. This one is usually used as a good measure of cycle length in the presence of noise (see Peters, 1994). This statistic is defined as

$$
V_{n}=\frac{(R / S)_{n}}{\sqrt{n}}
$$

A plot of $V_{n}$ versus $\log (n)$ would be flat if the system of random variables was an independent system, i.e. the $R / S$ statistic was scaling with the square root of time. For $H>0.5$ (persistent) the $R / S$ was scaling faster than the square root of time so the plot would be upward sloping. On the other hand for $H<0.5$ (antipersistent) the graph would have downward sloping.

The data used in the analysis are generated by the model outlined above in Sections 2 and 3 . In all cases, we used twenty trader types, that have the trend $g_{h}$ and bias $b_{h}$ generated by a random numbers generator for the normal distribution with parameters $N \sim(0,0.4)$ and $N \sim(0,0.3)$. The model generates four thousand observations, but to avoid problems with transients we do not use the first four hundred observations. The intensity of choice $(\beta=80)$ is the same for all simulations. Memory lengths or trading horizons are also generated by three distributions (normal, uniform, Weibull), the fourth is a given, fixed, value for all traders each in a particular simulation. With each predictor memory distribution, we have made four simulations with different expected memory lengths as follows $(5,10,20,40)$. To minimize a linear dependency of raw returns, which can bias estimates of the Hurst exponent significantly (see Peters, 1994), the AR(1) process representing residuals of the generated time series is used. This procedure, sometimes called prewhitening, usually eliminating a serial correlation, is the integral part of the $R / S$ analysis. Results of our experiments are demonstrated in Tables $1-4$.

In these tables there are two estimates of the Hurst exponent. The first one used data from period $n=9$ to 1800 . The second one (i.e. the Hurst mod) used data from period $n=9$ to break-even point of the $V$-statistics. Figures $1-4$ demonstrate $R / S$ and $V$-statistic for four different memory distributions with the same expected memory lengths $(E[\eta]=20)$ (see Table 3). There are visible differences in the position of maximal value of the $V$-statistic (break-even point). As a good example of the influence by the memory distribution are Figures 1-4. In Figure 1 (uniform distribution) the break-even point of the $V$-statistics is at the period length $n=20$ in comparison to the Figure 2 (normal distribution), where this situation occurs for $n=40$. Impact of different memory lengths is shown in Tables $1-4$, second column, where, for example, for the normal memory distribution, there is obvious the importance of the memory length for the pricing behaviour of the market. The longer traders' memory length the higher value of the period $n$ occurs (the break-even point in $V$-statistic). This rule does not hold for the Weibull memory distribution (see Table $1-3$ ). 
Figure 1

$R / S$ Analysis and $V$ - Statistic for Memory Distribution $N(20,5)$
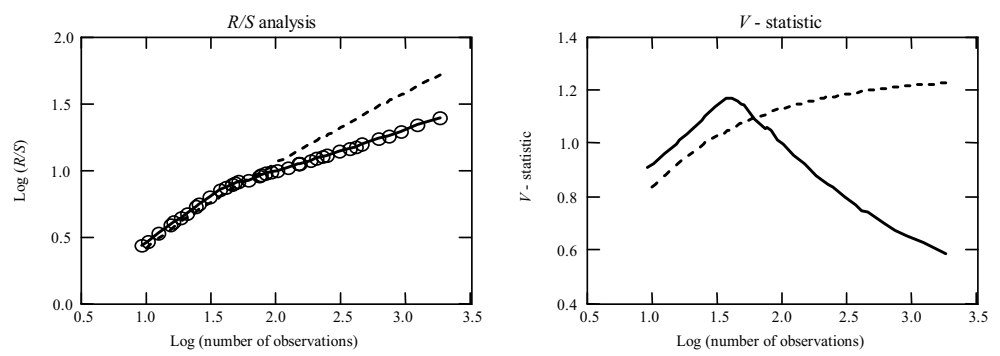

Figure 2

$R / S$ Analysis and $V$ - Statistic for Memory Distribution Uniform $(1,40)$
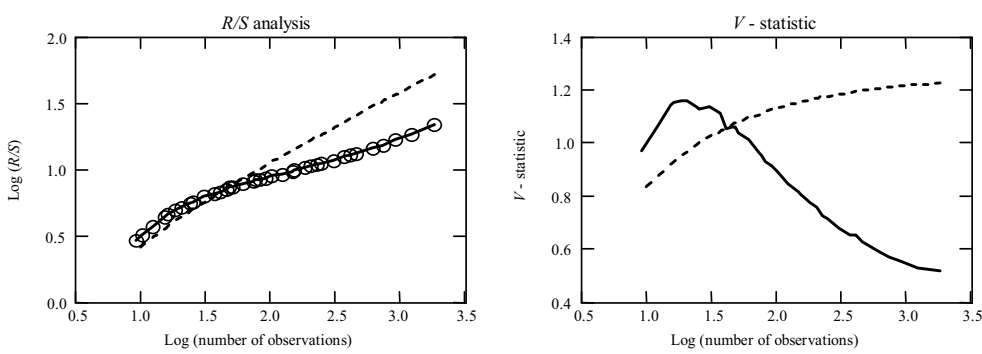

Figure 3

$R / S$ Analysis and $V$ - Statistic for memory Distribution Fixed (20)
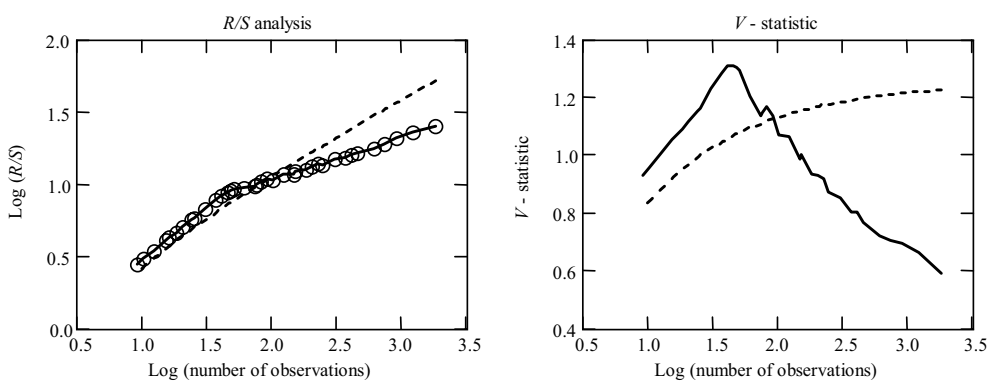

Figure 4

$R / S$ Analysis and $V$ - Statistic for Memory Distribution Weibull (1.3), $E=20$
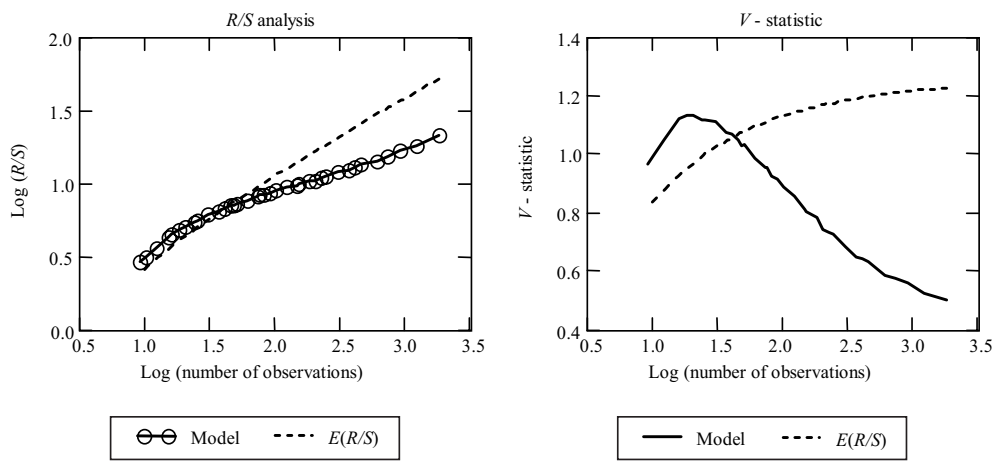
Table 1

Estimated Hurst Coefficients and Statistic of Raw Returns for Memory Mean $\mathbf{5}$

\begin{tabular}{|l|c|c|c|c|}
\hline$E(\eta)=5$ & Norm $(5,1.25)$ & Uniform $(1,10)$ & Fixed (5) & Weibull (1.3) \\
\hline Hurst & 0.112 & 0.122 & 0.171 & 0.128 \\
\hline Hurst mod & $0.858(9-20)$ & $0.636(9-20)$ & $0.851(9-20)$ & $0.542(9-25)$ \\
\hline $\operatorname{Var}(x)$ & 0.044 & 0.071 & 0.048 & 0.069 \\
\hline Kurtosis $(x)$ & 0.029 & 0.119 & -0.319 & 0.93 \\
\hline Skewness $(x)$ & 0.025 & 0.197 & -0.315 & -0.378 \\
\hline
\end{tabular}

Table 2

Estimated Hurst Coefficients and Statistic of Raw Returns for Memory Mean 10

\begin{tabular}{|l|c|c|c|c|}
\hline$E(\eta)=10$ & Norm $(10,2.5)$ & Uniform $(1,20)$ & Fixed (10) & Weibull (1.3) \\
\hline Hurst & 0.186 & 0.164 & 0.239 & 0.146 \\
\hline Hurst mod & $0.718(9-36)$ & $0.727(9-20)$ & $0.78(9-36)$ & $0.59(9-25)$ \\
\hline Var $(x)$ & 0.019 & 0.029 & 0.00584 & 0.027 \\
\hline Kurtosis $(x)$ & 0.642 & 1.915 & 0.371 & 1.379 \\
\hline Skewness $(x)$ & 0.186 & 0.41 & 0.096 & -0.163 \\
\hline
\end{tabular}

Table 3

Estimated Hurst Coefficients and Statistic of Raw Returns for Memory Mean 20

\begin{tabular}{|l|c|c|c|c|}
\hline$E(\eta)=20$ & Normal $(20,5)$ & Uniform $(1,40)$ & Fixed $(20)$ & Weibull (1.3) \\
\hline Hurst & 0.387 & 0.31 & 0.383 & 0.318 \\
\hline Hurst mod & $0.677(9-40)$ & $0.732(9-20)$ & $0.7(9-45)$ & $0.733(9-18)$ \\
\hline Var $(x)$ & 0.00000438 & 0.00732 & 0.0000515 & 0.00778 \\
\hline Kurtosis $(x)$ & 1.158 & 8.811 & 3.66 & 19.54 \\
\hline Skewness $(x)$ & -0.571 & -1.339 & -0.155 & 1.671 \\
\hline
\end{tabular}

Table 4

Estimated Hurst Coefficients and Statistic of Raw Returns for Memory Mean $\mathbf{4 0}$

\begin{tabular}{|l|c|c|c|c|}
\hline$E(\eta)=40$ & Normal $(40,10)$ & Uniform $(1,80)$ & Fixed $(40)$ & Weibull (1.3) \\
\hline Hurst & 0.462 & 0.421 & 0.47 & 0.399 \\
\hline Hurst mod & $0.611(9-72)$ & $0.556(9-72)$ & $0.60(9-72)$ & $0.587(9-45)$ \\
\hline Var $(x)$ & 0.00000398 & 0.0052 & 0.0000035 & 0.00267 \\
\hline Kurtosis $(x)$ & 0.245 & 24.652 & 0.203 & 17.795 \\
\hline Skewness $(x)$ & -0.503 & 2.328 & -0.895 & -2.392 \\
\hline
\end{tabular}

*

Short memories of predictors, i.e. short agent's investment horizons cause more volatile price realizations on the capital markets, but by values of the Hurst coefficients, there exist possibilities of the price predictions due to the persistence of the fundamental strategy structures. Long memories of predictors, i.e. long agent's in- 
vestment horizons cause more stable behaviour of price realizations on the capital markets. These tables demonstrate dependencies among agent's investment horizons and both local randomness and global determinism.

The fractal market hypothesis is a more general notation than the efficient market hypothesis. The fractal market hypothesis and the efficient market hypothesis are equivalent in the break-even point of the $V$-statistics. Here are equivalent the Brownian motion and the fractional Brownian motion. Therefore financial markets are nonlinear systems with a fractal structure of agent's investment horizons. These markets are unpredictable in the long-term period, but predictable in the short-term period. The key features - the lengths of memory and probability distribution in memory - influence self-similarity properties in agent's investment horizons.

References

Brock, W. A. (2001), Growth Theory, Nonlinear Dynamics and Economic Modelling. New York: Edward Elgar.

Brock, W. A., Hommes, C. H. (1997), "A Rational Route to Randomness." Econometrica, 65(5), pp. 1059-1095.

(1998), "Heterogeneous Beliefs and Routes to Chaos in a Simple Asset Pricing Model." Journal of Economic Dynamics and Control, 22, pp. 1235-1274.

Chiarella, C. (1992), "The Dynamics of Speculative Behaviour." Annals of Operational Research, 37, pp. 101-123.

Chiarella, C., He, X. (2003), "Heterogeneous Beliefs, Risk and Learning in a Simple Asset Pricing Model with a Market Maker." Macroeconomic Dynamic, 7, pp. 503-533.

Gaunersdorfer, A. (2000), "Endogenous Fluctuations in a Simple Asset Pricing Model with Heterogeneous Agents." Journal of Economic Dynamics and Control, 24, pp. 799-831.

Grassberger, P., Procaccia, I. (1983), "Measuring the Strangeness of Strange Attactors." Physica 9D: pp. 189-208.

Haltiwanger, J., Waldman, M. (1985), "Rational Expectations and the Limits of Rationality: an Analysis of Heterogenity." American Economic Review, 75, pp. 326-340.

Lorenz, H. W. (1993), Nonlinear Dynamical Economics and Chaotic Motion. Berlin: Springer-Verlag. Lucas, R. E. (1978), “Asset Prices in an Exchange Economy." Econometrica, 46, pp. 1429-1445.

Peters, E. E. (1994), Fractal Market Analysis, Applying Chaos Theory to Investment and Economics. New York: John Wiley\&Sons, inc.

Schone, R. (1997), Economics Dynamics Phase Diagrams and their Economic Application. Cambridge: Cambridge University Press.

Tu, P. N. V. (1992), Dynamical Systems an Introduction with Applications in Economics and Biology. Berlin: Springer-Verlag.

Vácha, L., Vošvrda, M. (2003), "Learning in Heterogeneous Agent Model with the WOA." $6^{\text {th }}$ International Conference on Applications of Mathematics and Statistics in Economy. Banská Bystrica, pp. 199-203.

Vošvrda, M. (2001), "Bifurcation Routes in Financial Markets." Proceedings of the $19^{\text {th }}$ International Conference Mathematical Methods in Economics 2001 Hradec Králové, pp. 199-205.

Vošvrda, M., Vácha, L. (2002), "Heterogeneous Agent Model with Memory and Asset Price Behaviour." Proceedings of the $20^{\text {th }}$ International Conference Mathematical Methods in Economics Ostrava, pp. 273-282.

Zeeman, E. C. (1974), "The Unstable Behaviour of Stock Exchange." Journal of Mathematical Economics, 1, pp. 39-49.

Zhou, W. X., Sornette, D. (2004), "Predictability of Large Future Changes in Major Financial Indices" (http://xxxlanlgov/PS_cache/cond-mat/pdf/0304/0304601pdf, pp.41-28). 